Endogenous fibrinolytic potential in tissue-plasminogen activator-modified thromboelastography analysis is significantly decreased in dogs suffering from diseases predisposing to thrombosis

Spodsberg, Eva-Maria Hohneck; Wiinberg, Bo; Jessen, Lisbeth Rem; Marschner, Clara Büchner; Kristensen, Annemarie Thuri

Published in:

Veterinary Clinical Pathology

DOI:

10.1111/vcp. 12068

Publication date:

2013

Document version

Early version, also known as pre-print

Citation for published version (APA):

Spodsberg, E-M. H., Wiinberg, B., Jessen, L. R., Marschner, C. B., \& Kristensen, A. T. (2013). Endogenous fibrinolytic potential in tissue-plasminogen activator-modified thromboelastography analysis is significantly decreased in dogs suffering from diseases predisposing to thrombosis. Veterinary Clinical Pathology, 42(3), 281290. https://doi.org/10.1111/vcp.12068 


\title{
Endogenous fibrinolytic potential in tissue-plasminogen activator-modified thromboelastography analysis is significantly decreased in dogs suffering from diseases predisposing to thrombosis
}

\author{
Eva H. Spodsberg ${ }^{1}$, Bo Wiinberg ${ }^{2}$, Lisbeth R. Jessen ${ }^{1}$, Clara B. Marschner ${ }^{1}$, Annemarie T. Kristensen ${ }^{1}$ \\ ${ }^{1}$ Department of Clinical Veterinary and Animal Sciences, Faculty of Health and Medical Sciences, University Hospital for Companion Animals, University \\ of Copenhagen, Frederiksberg C, Denmark; and ²Biopharmaceuticals Research Unit, Novo Nordisk A/S, Måløv, Denmark
}

\author{
Key Words \\ Canine, fibrinolysis, kaolin-activated TEG, \\ native TEG, tissue-factor (TF)-activated TEG \\ Correspondence \\ Dr. Eva H. Spodsberg, University Hospital \\ for Companion Animals, Department of \\ Veterinary Clinical and Animal Sciences, \\ Faculty of Health and Medical Sciences, \\ University of Copenhagen, Dyrlægevej 16, \\ Frederiksberg C 1870, Denmark \\ E-mail: Evahs@sund.ku.dk
}

DOI:10.1111/vcp. 12068
Background: In people, studies have shown that resistance to fibrinolysis could be a contributing factor to thrombosis. Tissue-plasminogen-activated (t-PA) thromboelastography (TEG) has been used to evaluate endogenous fibrinolytic potential. In dogs, TEG has been used for the diagnosis of various hemostatic disorders, but studies evaluating fibrinolysis are limited. Investigations into the potential of t-PA-modified TEG to monitor endogenous fibrinolytic potential are lacking in both healthy dogs and dogs with diseases predisposing to development of thrombosis.

Objectives: The aim of this study was to compare 3 t-PA-modified TEG assays and compare the endogenous fibrinolytic potential in dogs suffering from diseases associated with thrombosis with a group of healthy dogs.

Methods: Three different TEG assays, such as native, tissue factor-activated, and kaolin-activated, were modified with t-PA and used to compare whole blood samples from 16 healthy control dogs and 20 diseased dogs.

Results: Thromboelastography lysis variables were significantly affected by addition of t-PA in all 3 assays. Lysis results in diseased dogs were comparable to those in healthy dogs prior to addition of t-PA. After addition of t-PA, lysis results were significantly decreased in the diseased group compared with healthy dogs. The lowest median lysis levels were found in dogs with systemic inflammation and protein-losing disorders.

Conclusion: Addition of t-PA activates fibrinolysis in TEG of blood from both healthy dogs and dogs with diseases predisposing to thrombosis. The significantly decreased fibrinolysis in diseased dogs suggests that this may be a potential prothrombotic risk factor in dogs.

\section{Introduction}

Fibrinolysis is an essential part of the hemostatic process protecting against permanent thrombotic occlusion of damaged vessels in which a clot has formed. The balance between coagulation and fibrinolysis determines the size, quality, rate of dissolution, and life span of the clot. ${ }^{1,2}$ A balanced and healthy fibrinolytic mechanism dissolves the occlusive thrombus before permanent tissue damage occurs. ${ }^{1}$ Fibrinolysis is initiated when plasminogen is converted to plasmin by one of several plasminogen activators, with tissue plasminogen activator (t-PA) as the main intravascular activa- tor. $^{2}$ Plasmin mediates degradation of fibrinogen or soluble fibrin resulting in the formation of fibrin or fibrinogen degradation products (FDPs). Plasmin also mediates degradation of cross-linked, insoluble fibrin to produce $\mathrm{D}$-dimers. As D-dimers result only from the degradation of cross-linked fibrin in a stabilized clot, they are, in contrast to other degradation products, specific for active coagulation and fibrinolysis. ${ }^{3}$ Both the FDP and D-dimer assays are plasma-based and the only tests routinely used to evaluate fibrinolysis in dogs.

In human medicine, D-dimer tests are useful for the clinical diagnosis of patients suspected of having 
deep-vein thrombosis (DVT) and patients with pulmonary thromboembolism (PTE). ${ }^{4-6}$ In patients with a low or moderate pretest probability of disease, a negative $\mathrm{D}$-dimer test result helps in ruling out venous thromboembolism. ${ }^{7}$ In veterinary medicine, D-dimer tests have been used in the diagnosis of canine thromboembolic diseases, mainly PTE, with a negative test being considered a strong indicator of the absence of thromboembolism. ${ }^{3,8}$

Several disease conditions have been associated with the development of thrombosis in dogs. ${ }^{9}$ These include immune-mediated hemolytic anemia (IMHA), ${ }^{10}$ protein-losing conditions, ${ }^{11-13}$ hyperadrenocorticism, ${ }^{14}$ neoplasias, ${ }^{15,16}$ parvovirus infection, ${ }^{17}$ sepsis, ${ }_{1}^{18}$ early disseminated intravascular coagulation (DIC), ${ }^{19}$ and congestive heart failure. ${ }^{20}$ The current understanding and ability to diagnose and monitor thrombotic disease including fibrinolysis are limited. Specifically, defects of the fibrinolytic system or resistance to fibrinolysis have not been investigated, as no standardized tests are available to measure the global endogenous fibrinolytic potential of whole blood and determined risk factors for thromboembolic disease in dogs. In people, plasminogenactivator-induced fibrinolysis has been evaluated by thromboelastography (TEG) in several studies. ${ }^{21-27}$ Fibrinolysis has recently been standardized by using t-PA-induced, tissue-factor (TF)-activated TEG in human samples, and has been reported to provide rapid assessment of the endogenous fibrinolytic potential of whole blood. ${ }^{21}$ In another study with t-PA-modified TEG performed on whole blood from children with noncatheter-related DVT, the authors concluded that decreased fibrinolytic activity likely is a contributing factor to thrombosis. ${ }^{22}$ TEG is increasingly used in the diagnosis and monitoring of hemostatic disorders in dogs, ${ }^{28}$ including both hypercoagulable $\mathrm{e}^{10-20}$ and fibrinolytic disorders. ${ }^{25,26,29-31}$ Recently, TEG was used to measure TF-initiated fibrin formation and t-PA-induced fibrinolysis in human and canine hemophilic blood after addition of the anti-fibrinolytic Solulin, a soluble analog of thrombomodulin with anti-fibrinolytic properties used to increase clot lysis time. ${ }^{25}$ Blood obtained from hemophilic dogs given Solulin intravenously was found to generate clots that are resistant to fibrinolysis.

Studies assessing the ability of t-PA-modified TEG to evaluate fibrinolytic potential in healthy dogs and dogs with diseases known to predispose to thrombosis have not been reported. Early identification of dogs with altered fibrinolytic activity could ultimately help direct therapeutic measures or predict outcome. Therefore, our main hypothesis was that the addition of t-PA to TEG would activate fibrinolysis in canine whole blood from healthy dogs and dogs with diseases associated with development of thrombosis. In addition, we hypothesized that the fibrinolytic activity would be decreased in dogs with diseases associated with thrombosis when compared with healthy dogs. For that purpose, 3 different t-PA-modified TEG assays were compared.

\section{Materials and Methods}

The study was performed as a prospective, ex vivo study between January 2010 and March 2011 at the University Hospital for Companion Animals, Department of Veterinary Clinical and Animal Sciences (UHCA-DVCAS), Faculty of Health and Medical Sciences, University of Copenhagen, Denmark. The study was approved by the Ethics and Administrative Committee. All laboratory analyses were performed at The Central Laboratory, DVCAS. Forty dogs were included in this study, with a control group consisting of 20 healthy dogs and a group of 20 dogs with diseases potentially associated with thrombosis.

\section{Animals}

Healthy dogs: Fasting blood samples from 20 clinical healthy staff- or student-owned dogs were collected. Included were clinically healthy dogs of any breed and sex, of a minimum age of one year and a minimum weight of $10 \mathrm{~kg}$ to ensure that collected blood volume was $<2 \%$ of the total blood volume. The dogs were considered healthy based on absence of clinical signs of illness, an unremarkable physical examination and variables of a $\mathrm{CBC}$, biochemistry profile, a routine coagulation profile including APTT, PT, fibrinogen, D-dimers, TEG, and a urinalysis within the respective reference intervals (RI). Dogs with a history of receiving any medication during the preceding 2 weeks as well as bitches in estrus were excluded.

Diseased dogs: Blood samples from 20 clientowned dogs with disease conditions predisposing to thrombosis were collected between February 2010 and March 2011. Inclusion criteria for dogs with diseases associated with thromboembolic disease as defined by the literature ${ }^{9-20}$ were dogs of any breed and sex with a minimum age of one year and a minimum weight of $10 \mathrm{~kg}$ suffering from either (1) systemic inflammatory conditions (eg, acute pancreatitis, hepatitis, pneumonia, pyometra) resulting in systemic inflammatory response syndrome (SIRS) and confirmed by the combined results of the clinical examination (eg, hyperthermia or hypothermia, tachycardia, tachypnea), a moderate to marked increase in the acute-phase 
protein C-reactive protein (CRP, RI $0-35 \mathrm{mg} / \mathrm{L}$ ), and neutrophilia or neutropenia; (2) protein-losing conditions (eg, protein-losing enteropathy [PLE], proteinlosing nephropathy [PLN], vasculitis), based on results of the clinical examination and hypoalbuminemia (RI 26-44 g/L), either alone or in combination with panhypoproteinemia (total protein RI 57-82 g/L), in addition to a urine protein:creatinine (UPC) ratio of $>1$ in cases of PLN; (3) Diabetes mellitus, confirmed by hyperglycemia, hyperfructosaminemia and glucosuria, with or without ketonuria; (4) Hyperadrenocorticism confirmed by the combined results of the clinical and laboratory examinations $(\mathrm{CBC}$, biochemistry profile, urinalysis, ACTH response test, and/or low-dose dexamethasone suppression test); and (5) neoplasia, confirmed cytologically and/or histologically. Candidate dogs were identified based on history, clinical examination, and the above-mentioned criteria at admittance to the UHCA. All owners consented to their dogs being included in the study. Blood samples for CBC, biochemistry profile, a routine coagulation profile, and TEG were collected before treatment was initiated. Dogs with a history of medication with nonsteroidal anti-inflammatory drugs, corticosteroids, and anticoagulants during the preceding 2 weeks as well as bitches in estrus were excluded.

\section{Sampling}

Healthy dogs were fasted 12 hours prior to blood collection. From diseased dogs, blood was collected at admittance to the hospital. In both groups, blood was collected by jugular venipuncture, by applying minimum stasis and using a 21 -gauge butterfly needle. Blood was collected in the following order: one serum tube $(5 \mathrm{~mL}$, Vacuette, Greiner Bio-One International AG, Kremsmuenster, Austria), followed by 5 citrated plastic tubes (2.7 mL, 0.109 M, 3.2\%; BD Vacutainer TM Plus Plastic Citrate tube), followed by one EDTA tube ( $2 \mathrm{~mL}$, Vacuette, Greiner Bio-One International AG).

\section{Routine hematology, biochemistry, and coagulation tests}

After sampling, citrate tubes were carefully inverted 5 times to ensure mixing of citrate and blood, in a 1:9 ratio. One randomly selected citrate tube was used for the standard coagulation profile. Serum and citrate tubes for biochemistry and coagulation profiles were centrifuged at $4000 \mathrm{~g}$ for 120 seconds, and serum and plasma were separated and collected within $30 \mathrm{~min}-$ utes of sampling. The $\mathrm{CBC}$ and biochemistry profile were run within 2 hours of sampling. The CBC was determined using an ADVIA 2120 automated hematology analyzer (Siemens Health Care Diagnostics Inc., Deerfield, IL, USA) and the blood smear was evaluated by a clinical pathologist. The biochemistry profile was analyzed using an ADVIA 1800 (Siemens Health Care Diagnostics Inc.). A coagulation profile was performed immediately or the plasma was stored at $-80^{\circ} \mathrm{C}$ until analysis. APTT (aPTT SynthAFax; Instrumentation Laboratory, Bedford, MA 01730, USA), PT (PT-Fibrinogen, Instrumentation Laboratory), and fibrinogen concentration (PT-Fibrinogen, Instrumentation Laboratories) were measured using the ACL TOP 500 analyzer (Instrumentation Laboratory). D-dimer concentration was measured with an immunofiltration assay (D-dimer Single Test) on the NycoCard READER II (Medinor A/S, Brøndby, Denmark) found to give accurate results for canine samples. ${ }^{32}$ A plasma pool from 10 healthy dogs was used as a reference for plasma-based coagulations assays. The plasma pool was stored at $-80^{\circ} \mathrm{C}$ for no longer than 6 months. The remaining citrate tubes were stored in an upright position in a tube rack at room temperature $\left(20-25^{\circ} \mathrm{C}\right)$ for 30 minutes for subsequent TEG analyses.

\section{TEG analysis}

Analyses using native, TF-activated, and kaolinactivated assays, respectively, with or without t-PA added, were performed using a computerized thromboelastograph (TEG 5000 Haemostasis Analyzer, Haemonetics Corporation, Braintree, MA, USA). Blood samples were analyzed in duplicate for healthy dogs resulting in a total of 12 TEG analyses per animal. For the diseased dogs, single measurements were performed.

The analyses were performed using preheated recalcified TEG cups $\left(37^{\circ} \mathrm{C}, 20 \mu \mathrm{L} 0.2 \mathrm{M} \mathrm{CaCl}_{2}\right)$ prior to addition of citrated whole blood (WB). For native analysis, $340 \mu \mathrm{L}$ citrated WB was added directly to the cups. For the TF-activated samples, citrated whole blood was activated with a solution of human recombinant TF (Innovin, Siemens Healthcare Diagnostics, Deerfield, IL, USA) at a final dilution of 1:50.000, as previously described. ${ }^{32}$ Kaolin activation was initiated by adding $1 \mathrm{~mL}$ citrated WB to kaolin-primed tubes (Haemonetics Corporation), and $340 \mu \mathrm{L}$ of this mixture was added to the TEG cups. TEG analyses were run for 120 minutes at $37^{\circ} \mathrm{C}$. Six TEG parameters were collected in the present study: the reaction time (R), the clot formation time $(\mathrm{K})$, the angle $(\alpha)$ representing the kinetics of fibrin production and cross-linking, the maximum amplitude (MA) reflecting the maximal clot 
strength, and the percent clot lysis detected at 30 minutes (Ly30) and 60 minutes (Ly60), respectively, after MA is reached and representing the fibrinolytic activity.

A pilot study was performed on WB samples from healthy dogs with the addition of t-PA (Single-chain recombinant tissue plasminogen activator $10 \mathrm{ng} / \mu \mathrm{L}$, Bionordika A/S, Glostrup, Denmark). A sample of $5 \mu \mathrm{L}$ t-PA was prediluted 1:10 in a 4-(2-hydroxyethyl)-1piperazine ethanesulfonic acid (HEPES) buffer with $2 \%$ bovine serum albumin (BSA). Samples of $2.5 \mu \mathrm{L}$, $5.0 \mu \mathrm{L}, 7.5 \mu \mathrm{L}$, or $10 \mu \mathrm{L}$ of the prediluted t-PA were mixed with $430 \mu \mathrm{L}$ HEPES-BSA. An aliquot of $25 \mu \mathrm{L}$ of this mixture was added to $400 \mu \mathrm{L}$ citrated WB to give increasing final concentrations of t-PA $(3.4 \mathrm{pg} / \mu \mathrm{L}$, $6.7 \mathrm{pg} / \mu \mathrm{l}, 10.0 \mathrm{pg} / \mu \mathrm{L}$ and $13.4 \mathrm{pg} / \mu \mathrm{L}$ ) in a total assay volume of $340 \mu \mathrm{L}$. Higher t-PA concentrations showed higher inhibitory effect on clotting activity and more rapid completion of fibrinolysis. A final concentration of $10.0 \mathrm{pg} / \mu \mathrm{L} \mathrm{t}-\mathrm{PA}$ resulted in consistent and maximal fibrinolysis without affecting TEG-parameters R, K, $\alpha$, and MA when compared with TEG without addition of t-PA, and thus used for the study (data not shown).

For the TF-activated assay, $7.5 \mu \mathrm{L}$ prediluted t-PA was mixed with $430 \mu \mathrm{L}$ diluted TF, and $25 \mu \mathrm{L}$ of the TF-t-PA mixture was added to $400 \mu \mathrm{L}$ citrated WB. $340 \mu \mathrm{L}$ of the TF-t-PA-WB mixture was added to the TEG cups yielding a final TF:WB ratio of 1:50.000. For the kaolin-activated assay, $7.5 \mu \mathrm{L}$ prediluted t-PA was mixed with $430 \mu \mathrm{L}$ HEPES-BSA buffer, and $62.5 \mu \mathrm{L}$ of the prediluted t-PA was then mixed with the kaolinWB mixture. $340 \mu \mathrm{L}$ of the kaolin-t-PA-WB mixture

Table 1. Basic data including fibrinogen, D-dimers, platelet counts, and hematocrit in 16 control dogs and 20 diseased dogs predisposed to thrombosis.

\begin{tabular}{|c|c|c|c|c|c|c|c|c|}
\hline Dog \# & Breed & $\begin{array}{l}\text { Age } \\
\text { (years) }\end{array}$ & Sex & Clinical Status/Diagnosis & $\begin{array}{c}\text { Fibrinogen } \\
\text { (g/L) }\end{array}$ & $\begin{array}{l}\text { D-Dimer } \\
\text { (mg/L) }\end{array}$ & $\begin{array}{l}\text { Platelets } \\
\left(10^{9} / \mathrm{L}\right)\end{array}$ & $\begin{array}{l}\text { Hematocrit } \\
(\%)\end{array}$ \\
\hline \multicolumn{9}{|c|}{ Controls } \\
\hline \multirow[t]{4}{*}{$n=16$} & Different breeds & $1-7$ & $7 \mathrm{~F}$ & Clinically healthy & Range 1-4 & Range $0.0-0.5$ & Range 200-500 & Range 39-59 \\
\hline & & & $5 \mathrm{FS}$ & & & & & \\
\hline & & & $1 \mathrm{M}$ & & & & & \\
\hline & & & $3 \mathrm{MC}$ & & & & & \\
\hline \multicolumn{9}{|c|}{ Dogs suffering from diseases predisposing to thrombosis } \\
\hline 1 & German Shepherd & 5 & M & Acute myocarditis & 8.02 & 0.1 & 99 (Aggregates) & 49 \\
\hline 2 & French Bulldog & 1 & M & $\begin{array}{l}\text { Obstructive foreign body } \\
\text { intestine }\end{array}$ & 6.76 & NA & 323 & 58 \\
\hline 3 & Island Sheepdog & 12 & $\mathrm{~F}$ & Severe mastitis. SIRS & 12.7 & 0.2 & NA & NA \\
\hline 4 & Mixed breed & NA & $\mathrm{F}$ & $\begin{array}{l}\text { Severe inflammation paw. } \\
\text { SIRS }\end{array}$ & 11.6 & 0.2 & 463 & 43 \\
\hline 5 & Australian Kelpie & 9 & $\mathrm{~F}$ & Pyometra. SIRS & 3.84 & 0.8 & 394 & 45 \\
\hline 6 & Labrador Retriever & 11 & $\mathrm{~F}$ & Pyometra. SIRS & 6.81 & 0.3 & 589 & 58 \\
\hline 7 & Labrador Retriever & 4 & $\mathrm{~F}$ & Acute mild pancreatitis & 5.37 & 0.7 & 247 & 53 \\
\hline 8 & Mixed breed & 6 & $\mathrm{~F}$ & Pneumonitis. SIRS. & $4.92-5.42$ & 0.3 & 219 & 58 \\
\hline 9 & Basenji & 12 & M & PLN & 4.02 & 0.5 & 720 & 48 \\
\hline 10 & Bichon Havanais & 3 & M & LPE. PLE & NA & NA & 696 & 46 \\
\hline 11 & Boxer & 8 & MC & Chronic LPE. PLE & 9.14 & 0.2 & 309 & 52 \\
\hline 12 & Mixed breed & 7 & FS & $\begin{array}{l}\text { Chronic necrotizing } \\
\text { pancreatitis. Vasculitis. } \\
\text { SIRS. }\end{array}$ & 4.39 & 0.7 & 285 & 40 \\
\hline 13 & Kleiner Münsterländer & 6 & $\mathrm{MC}$ & PLN. Renal amyloidosis & 10.88 & NA & 240 & 32 \\
\hline 14 & German Shepherd & 9 & FS & $\begin{array}{l}\text { Splenic+hepatic } \\
\text { hemangiosarcoma }\end{array}$ & 0.8 & 4.1 & 55 (Aggregates) & 18.5 \\
\hline 15 & Cairn Terrier & 12 & FS & Primary thrombocytosis & NA & 0.7 & 1206 & 37 \\
\hline 16 & English Bulldog & 1 & $\mathrm{MC}$ & Leukemia & 2.31 & 1.6 & 87 (No aggregates) & 37 \\
\hline 17 & Cocker Spaniel & 11 & $\mathrm{~F}$ & Mammary carcinoma & 3.22 & NA & 905 & 31 \\
\hline 18 & Bernese Mountain Dog & 8 & M & $\begin{array}{l}\text { Primary histiocytic } \\
\text { sarcoma }\end{array}$ & 4.81 & 2.6 & 92 (No aggregates) & 42 \\
\hline 19 & Danish-Swedish Farmdog & 6 & FS & Diabetic ketosis & 4.51 & $<0.1$ & 461 & 48 \\
\hline 20 & Labrador Retriever & 6 & M & Hepatic cirrhosis. SIRS. & 1.44 & 0.2 & 218 & 41 \\
\hline
\end{tabular}

F indicates female, FS, female spayed, LPE, lymphocytic-plasmacytary enteritis, M, male, MC, male castrated, PLE, protein-losing enteropathy, PLN, protein-losing nephropathy, SIRS, systemic inflammatory response syndrome, NA, not analyzed.

The bold numbers indicate values outside the reference range. 


\section{Ly30 + t-PA}

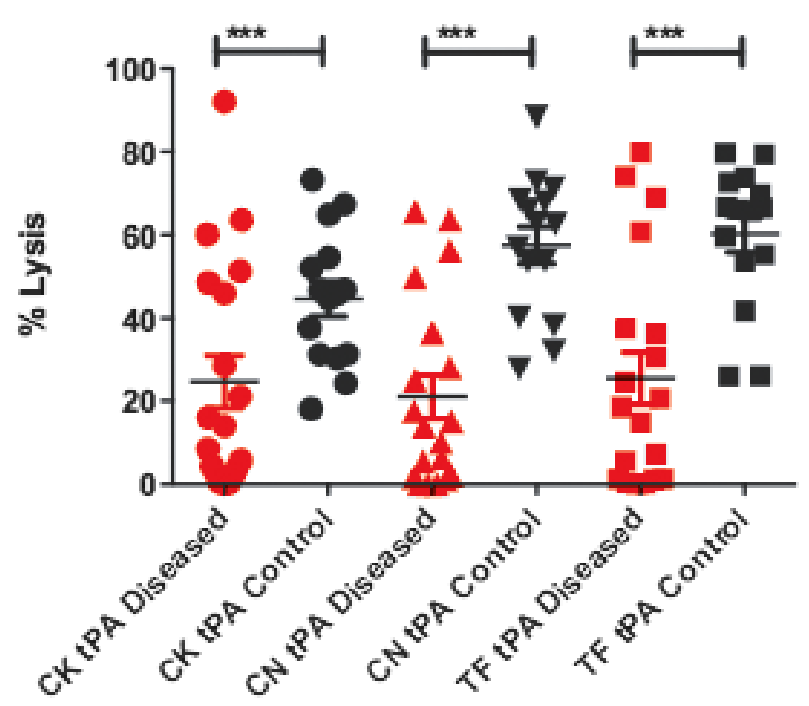

Figure 1. After the addition of tissue-plasminogen activator, percent clot lysis after 30 minutes (Ly30) was significantly lower in the diseased dogs compared with healthy dogs in all 3 assays, indicating a decreased fibrinolytic activity and potential predisposition to thrombosis. CK indicates Kaolin-activated assay; CN, citrated native-activated assay, TF, tissue-factor-activated assay. ${ }^{* * *}=P<.0001$

was added to the TEG cups, yielding the final t-PA concentration of $10 \mathrm{pg} / \mathrm{uL}$ in the cup.

\section{Statistical analysis}

Descriptive statistics were used to summarize dog characteristics at UHCA admission as well as findings of the clinical examination, CBCs, and serum biochemical analyses for dogs with disease conditions predisposing to thrombosis. GraphPad Prism 5 (GraphPad Software Inc., San Diego, CA, USA) was used for statistical analyses. To determine distribution of the data, D'Agostino-Paerson normality test was applied. For normally distributed data, repeated measures ANOVA was used followed by a Bonferroni multiple comparison posttest. For nonparametric data, a Friedmann test was used followed by a Dunns posttest. We used a Mann-Whitney test for comparison of the 2 unmatched groups. Statistical significance was set at $P<.05$. Coefficients of variation for the 3 types of assays were determined for the samples from the healthy dogs, as these were measured in duplicate.

\section{Results}

Gender, breed, age, individual final diagnosis, fibrinogen, D-dimer, platelet count, and HCT of all dogs are listed in Table 1. The control group consisted of 5 male (4 castrated males, 1 intact male) and 15 female dogs (10 intact females, 5 spayed females) with a mean age of 4.2 years (range 1.1-7.1 years). Of the 20 control dogs that were initially recruited, 3 dogs were excluded due to abnormal serum biochemistry results, and one dog was excluded due to a urinary tract infection. In one dog, the age was unknown. In another dog, hematology results were missing. In 2 dogs, results of a routine coagulation profile were missing, and in another 2 dogs, D-dimers could not be analyzed due to lipemia. Urinalysis was conducted as part of the diagnostic procedure in 15 of the dogs. No sampled animals were retrospectively excluded from the study.

Table 2. Results of 3 different thromboelastography assays without addition of tissue-plasminogen activator with blood from control dogs and diseased dogs predisposed to thrombosis.

\begin{tabular}{|c|c|c|c|c|c|c|}
\hline & \multicolumn{3}{|c|}{ Control Dogs } & \multicolumn{3}{|c|}{ Diseased Dogs } \\
\hline & CK & $\mathrm{CN}$ & TF & CK & $\mathrm{CN}$ & TF \\
\hline $\mathrm{R}$ (minutes) & $\begin{array}{l}5.1(3.3-7.9) \\
\text { (CV: 8.6\%) }\end{array}$ & $\begin{array}{l}6.1(3.9-11) \\
\text { (CV: 29.8\%) }\end{array}$ & $\begin{array}{l}5.3(3.7-7.6) \\
\text { (CV: 4.9\%) }\end{array}$ & $5(3.3-7.7)$ & $5.45(4-11.9)$ & $4.6(3.1-8.7)$ \\
\hline K (minutes) & $\begin{array}{l}1.9(1.2-2.8) \\
\text { (CV: } 20.4 \%)\end{array}$ & $\begin{array}{l}2.4(1.7-7.7) \\
(C V: 31.2 \%)\end{array}$ & $\begin{array}{c}1.7(1.4-2.8) \\
(C V: 11.5 \%)\end{array}$ & $1.3(0.8-5.5)$ & $1.4(1-5.8)$ & $1.15(0.8-6.5)$ \\
\hline Angle (deg) & $\begin{array}{c}63.1(45.6-71.9) \\
(\mathrm{CV}: 5.5 \%)\end{array}$ & $\begin{array}{c}54.1(29.3-65.7) \\
\quad(C V: 10.1 \%)\end{array}$ & $\begin{array}{c}62.7(52.9-70) \\
(\mathrm{CV}: 3.8 \%)\end{array}$ & $69.9(36.9-78.6)$ & $64(33.6-76.2)$ & $71.7(27.7-78.8)$ \\
\hline $\mathrm{MA}(\mathrm{mm})$ & $\begin{array}{c}58.6(48.5-66.7) \\
(\mathrm{CV}: 7 \%)\end{array}$ & $\begin{array}{c}56.9(42.5-65.8) \\
\text { (CV: } 7 \%)\end{array}$ & $\begin{array}{c}59(48.8-67.6) \\
(\mathrm{CV}: 2.3 \%)\end{array}$ & $71.9(36.8-83.9)$ & $69.8(35.4-71.7)$ & $73(32.9-73.7)$ \\
\hline Ly30 (\%) & $\begin{array}{c}0(0-5.7) \\
(\mathrm{CV}: 133.7 \%)\end{array}$ & $\begin{array}{c}0(0-4.8) \\
(\mathrm{CV}: 755.2 \%)\end{array}$ & $\begin{array}{c}0(0-6.7) \\
(\mathrm{CV}: 147 \%)\end{array}$ & $0(0-4.8)$ & $0(0-8.7)$ & $0(0-9.2)$ \\
\hline Ly60 (\%) & $\begin{array}{l}1.5(0-14.3) \\
\text { (CV: } 74.6 \%)\end{array}$ & $\begin{array}{c}0.8(0-11) \\
\text { (CV: 134.7\%) }\end{array}$ & $\begin{array}{l}1.1(0-12.8) \\
\text { (CV: 67.4\%) }\end{array}$ & $1.5(0-9.6)$ & $0.8(0-9.3)$ & $0.7(0-11.2)$ \\
\hline
\end{tabular}

Data are means (range) and CV in control dogs (duplicate assays).

CK indicates Kaolin-activated assay; CN, citrated native-activated assay, TF, tissue-factor-activated. 
Table 3. Thromboelastography results with addition of tissue plasminogen activator with blood of healthy control dogs and diseased dogs predisposed for thrombosis.

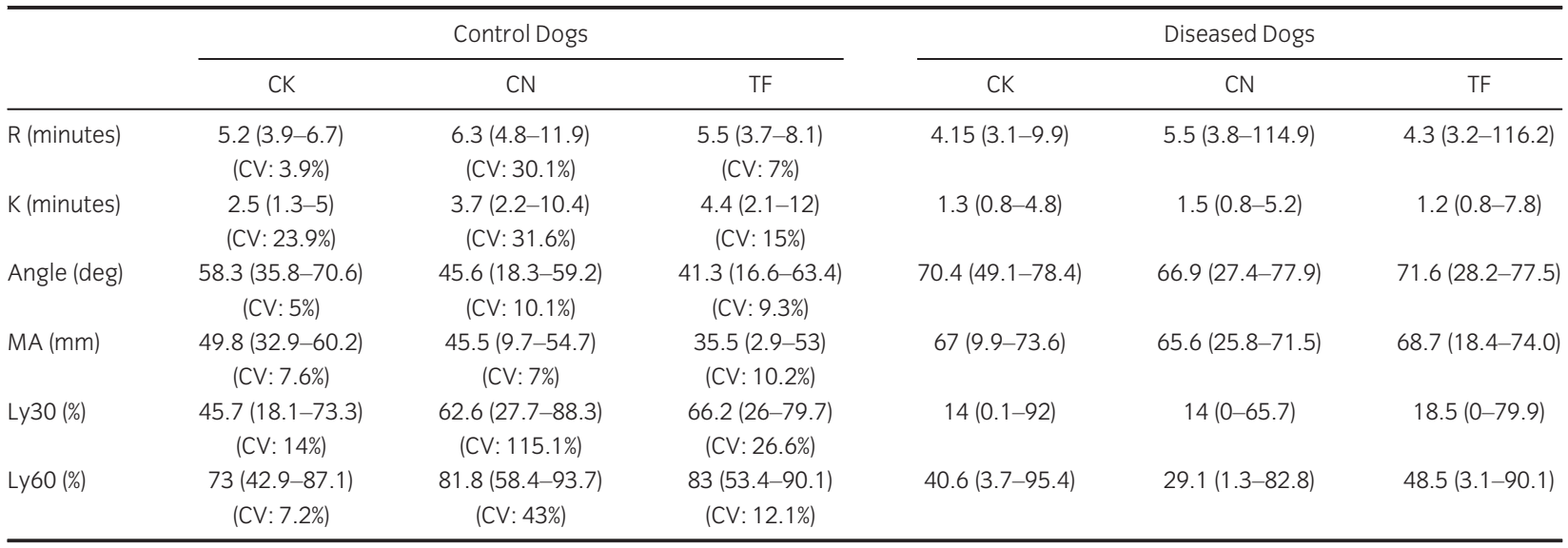

Data are means (range) with CV in control dogs (duplicate assays).

CK indicates Kaolin-activated assay; CN, citrated native-activated assay, TF, tissue-factor-activated.

Comparisons of Ly30 and Ly60 values before and after addition of t-PA in both groups of dogs were performed using Friedmann test and Dunn's post hoc tests. The Friedmann test showed highly significant differences in both healthy and diseased dogs (all $P<.0001$ ) when comparing the 3 different assays for Ly30 and Ly60, respectively. Dunn's post hoc test showed that addition of t-PA significantly decreased Ly30 and Ly60 measurements (all $P<.05$ ) in both healthy and diseased dogs, regardless of assay type (Figure 1).

There were no significant correlations between fibrinogen, D-dimer, platelet count, hematocrit, and lysis parameters Ly 30 and Ly60 before or after addition of t-PA (results not shown). TEG variables before and after addition of t-PA in both groups are reported in Tables 2 and 3. There were no significant differences in lysis parameters among the 3 assays or between the 2 groups of dogs before addition of t-PA.

The results of the Mann-Whitney test showed that Ly30 and Ly60 were significantly different after addition of t-PA (all $P<.05$ ) between healthy and diseased dogs, regardless of the assay, but with the highest significance $(P<.0001)$ in the native assay. Examples of a t-PA-modified TF-activated TEG tracing from a clinically healthy dog and a diseased dog with resistance to fibrinolysis are shown in Figure 2.

Threshold values for Ly30 and Ly60 for all 3 assays were defined as lysis values (\%) below the lower end of the range of the healthy control group. Depending on the assay used, between 11 and 13 of the 20 diseased dogs had Ly30 values below the ranges obtained from running the t-PA assays in healthy dogs. For Ly60, between 10 and 14 diseased dogs had values below the lower end of the range of the healthy control group depending on the assay.

$\mathrm{CV}_{\mathrm{A}}$ values were only obtained for healthy dogs based on duplicate analyses and are reported in Tables 2 and 3 before and after addition of $t-P A$, respectively. $\mathrm{CV}_{\mathrm{A}}$ before addition of t-PA was lowest in the TF-activated assays for all measured parameters except Ly30 and highest in the native assays for all measured parameters except MA. After addition of t-PA, the kaolin-activated assays had the lowest analytical variation for all measured parameters except K and MA.

\section{Discussion}

The results of this study show that t-PA-modified TEG is able to activate fibrinolysis in canine whole blood from both healthy dogs and dogs with diseases known to predispose to thrombosis. However, the diseased dogs were significantly more resistant to t-PA-induced fibrinolysis than the healthy control group.

The majority of dogs in the diseased group had lysis values significantly below the values obtained from healthy dogs. Although there was an overlap between some of the dogs in the diseased group and the healthy dogs, a substantial number of diseased dogs were clearly resistant to activation of fibrinolysis, independent of the assay used. Interestingly, dogs with fibrinolytic activity below the lower range of healthy dogs in all 3 assays after the addition of t-PA had all been diagnosed with either a systemic inflammatory condition or a protein-losing disorder. The lowest individual lysis variables were observed in dogs with PLN, necrotizing pancreatitis, SIRS and hepatic cirrhosis, 

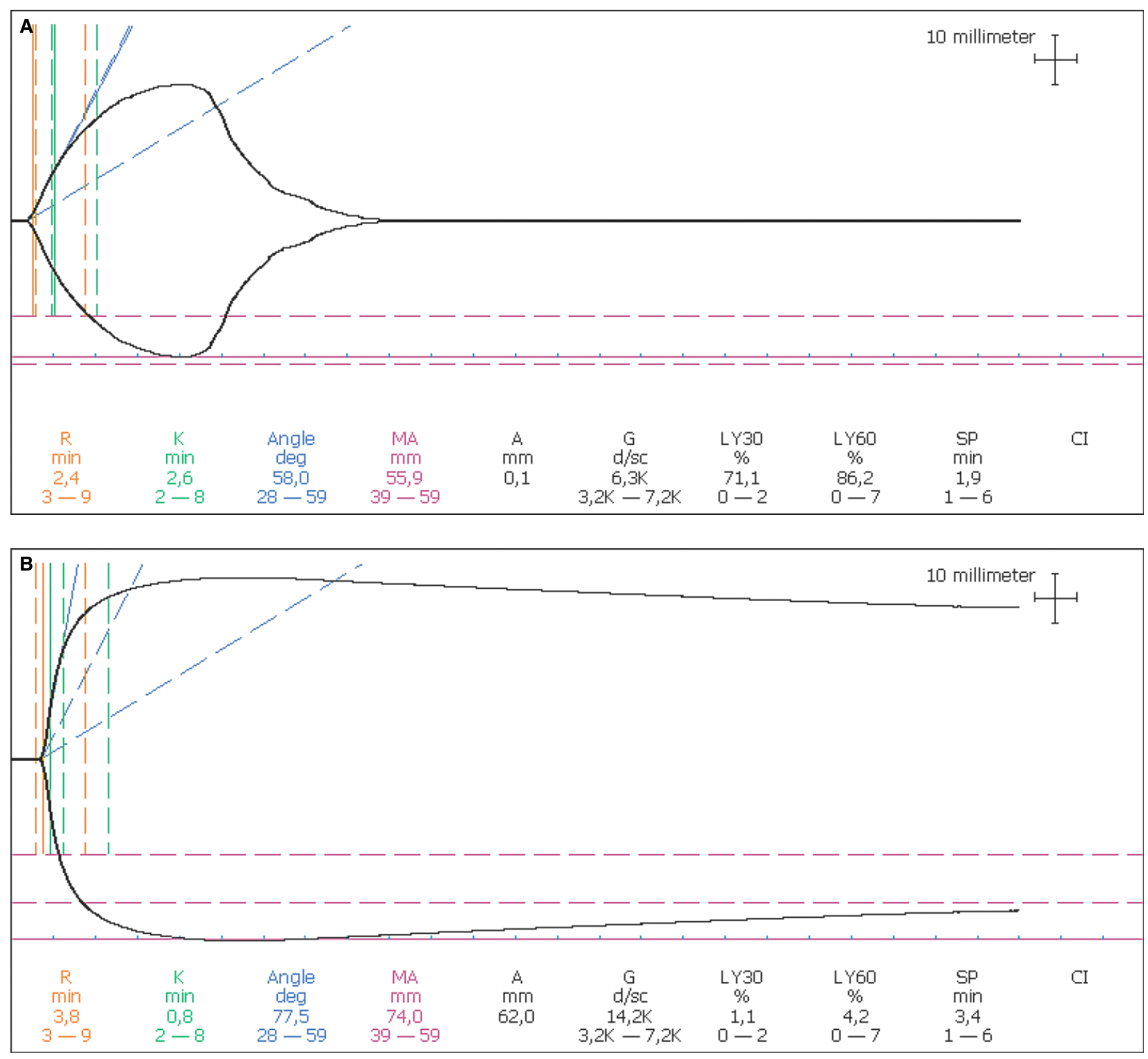

Figure 2. Examples of tissue-plasminogen-modified (t-PA) tissue-factor-activated thromboelastography tracings from (A) a clinically healthy dog where addition of $10.0 \mathrm{pg} / \mu \mathrm{L} \mathrm{t}-\mathrm{PA}$ resulted in a clearly visible activation of fibrinolysis; (B) from a diseased dog predisposed to thrombosis, where the resistance to fibrinolytic activity in spite of addition of $10.0 \mathrm{pg} / \mu \mathrm{L} \mathrm{t}-\mathrm{PA}$ is clearly visible. R indicates reaction time in minutes, and represents the time span from initiation until the first fibrin polymers are produced. The cut-off value is when the amplitude reaches $2 \mathrm{~mm}$. $\mathrm{K}=$ clot formation time in minutes, measured from the end of $\mathrm{R}$ until an amplitude of $20 \mathrm{~mm}$ is reached, representing the speed of clot formation. $\mathrm{A}=$ angle in degrees, representing the kinetics of fibrin production and cross-linking. MA = maximum amplitude reached in millimeters, reflecting the maximal clot strength. Ly30 = clot lysis in percent at 30 minutes after MA is reached; Ly60:clot lysis in percent at 60 minutes after MA is reached.

with SIRS due to pneumonitis, and mammary carcinoma, respectively.

As the majority of dogs with decreased fibrinolytic activity suffered from conditions associated with systemic inflammation, inhibition of fibrinolysis secondary to acute inflammation could be one of the major reasons for our findings. Acute inflammation activates systemic coagulation by TF-mediated thrombin generation, downregulation of physiological anticoagulant mechanisms, and inhibition of fibrinolysis, resulting in enhanced fibrin formation and impaired fibrin degradation. ${ }^{33}$

Sepsis has previously been associated with reduced activity of endogenous anticoagulants in dogs, suggesting an imbalance of hemostasis with a tendency toward a procoagulant state in affected dogs. ${ }^{18}$ For instance, significantly increased thrombin-activatable fibrinolysis inhibitor (TAFI) activity was determined in dogs with bacterial sepsis. ${ }^{34}$ 
Hemostatic abnormalities consistent with systemic hypercoagulability are common in dogs with PLE and PLN. ${ }^{11,35}$ PLN was associated with an increased risk of thromboembolism in $22.2 \%$ of dogs in a case series of 137 dogs. ${ }^{36}$ The mechanisms leading to hypercoagulability and increased risk for thrombosis in dogs with protein-losing conditions are multifactorial; one of the proposed causes is enteric and renal loss of antithrombin (AT) as dogs with PLE and PLN have decreased AT levels. ${ }^{1,35} \mathrm{AT}$ deficiency could lead to an increased amount of thrombin, resulting in hypercoagulability. ${ }^{11}$ Endothelial cell injury with secondary activation of the coagulation cascade and urinary loss of fibrinolytic and anticoagulant proteins are other possible causes. ${ }^{35}$

We used and compared 3 different TEG assays in our study. For the native assay, recalcified citrated whole blood was used without an activator, and as such carrying a higher risk of analytical variation. ${ }^{32}$ In our study, the native assay had the highest analytical variation both with and without addition of t-PA. For the TF-activated assay, we used human recombinant TF as an activator to initiate coagulation through the extrinsic pathway in a concentration validated earlier. $^{32}$ The TF-activated assay had the lowest CV values without addition of t-PA. Activation with kaolin initiates coagulation via the intrinsic pathway. The kaolinactivated assay had the lowest analytical variation after addition of t-PA. All 3 assays can be used for activation of fibrinolysis with the addition of t-PA. However, the results cannot be used interchangeably as the activated assays produced the least variable and most reproducible results, while the unactivated citrated native assay showed high analytical variation as seen in other canine studies. ${ }^{11,32}$ A study of 3 different TEG assays (native, TF, kaolin) on WB from clinically healthy cats confirmed the native assay having the highest CV and the kaolin assay having the lowest CV. ${ }^{37}$ In human samples, a CV between $4 \%$ and $14 \%$ with no significant differences between TF- and kaolin-activated TEG assays was described. ${ }^{38}$ The large analytical variation in the native assay compared with the activated assays can be explained by the absence of a standardized amount of activator as initiation of coagulation in the native assay occurs spontaneously. ${ }^{37}$

As all TEG parameters were significantly affected in all 3 assays by the addition of t-PA in the healthy control group, standard TEG analyses cannot be replaced by t-PA-modified TEG to evaluate individual hemostatic abnormalities besides fibrinolysis.

A limitation of this study was the small number of dogs with diseases predisposing to thrombosis. Further evaluation of fibrinolysis should include a larger group of dogs, with different disease categories to study and compare the levels of t-PA-activated fibrinolysis and their potential correlation with development of thrombosis. In addition, measurement of downregulators of fibrinolysis may be useful in evaluating the cause for the decreased fibrinolytic response in dogs with diseases predisposing to thrombosis.

Addition of t-PA to TEG can be used as a tool for the assessment of endogenous fibrinolytic potential in healthy dogs and dogs with diseases associated with thrombosis. Furthermore, many dogs suffering from diseases associated with thrombosis are more resistant to t-PA than healthy controls. Dogs with the highest resistance to t-PA in the present study were either suffering from systemic inflammation or a protein-losing disease. Thus, t-PA-modified TEG may be a promising tool for the detection of decreased endogenous fibrinolytic potential or resistance to fibrinolysis.

Activated TEG assays appear to be less variable and more reproducible compared with the native assay in this study and therefore, activated assays are recommended for future studies.

The clinical consequences of decreased fibrinolytic activity are currently not known. Further investigation of decreased fibrinolytic activity in dogs may help elucidate the complex pathophysiological mechanisms leading to hemostatic dysfunction in disease and the potential development of thrombosis in dogs.

\section{Acknowledgment}

The authors thank Annette U. Martinsen for excellent technical assistance.

Disclosure: The authors have indicated that they have no affiliations or financial involvement with any organization or entity with a financial interest in, or in financial competition with, the subject matter or materials discussed in this article.

\section{References}

1. Gorog DA. Prognostic value of plasma fibrinolysis activation markers in cardiovascular disease. J Am Coll Cardiol. 2010;55:2701-2709.

2. Stokol T. Plasma D-dimer for the diagnosis of thromboembolic disorders in dogs. Vet Clin North Am Small Anim Pract. 2003;33:1419-1435.

3. Nelson OL, Andreasen C. The utility of plasma D-dimer to identify thromboembolic disease in dogs. J Vet Intern Med. 2003;17:830-834.

4. Wells PS, Anderson DR, Rodger M, et al. Evaluation of D-dimer in the diagnosis of suspected deep-vein thrombosis. N Engl J Med. 2003;349:1127-1135. 
5. Kelly J, Rudd A, Lewis RR, Hunt BJ. Plasma D-dimers in the diagnosis of venous thromboembolism. Arch Intern Med. 2002;162:747-756.

6. Mountain D, Jacobs I, Haig A. The VIDAS D-dimer test for venous thromboembolism: a prospective surveillance study shows maintenance of sensitivity and specificity when used in normal clinical practice. Am J Emerg Med. 2007;25:464-471.

7. Bates SM, Grand'Maison A, Johnston M, Naguit I, Kovacs MJ, Ginsberg JS. A latex D-dimer reliably excludes venous thromboembolism. Arch Intern Med. 2001;161:447-453.

8. Rosser EJ Jr. Use of the D-dimer assay for diagnosing thrombosis in cases of canine cutaneous vasculitis. Vet Dermatol. 2009;20:586-590.

9. Goggs R, Benigni L, Fuentes VL, Chan DL. Pulmonary thromboembolism. J Vet Emerg Crit Care (San Antonio). 2009; 19:30-52.

10. Fenty RK, Delaforcade AM, Shaw SE, O`Toole TE. Identification of hypercoagulability in dogs with primary immune-mediated hemolytic anemia by means of thromboelastography. J Am Vet Med Assoc. 2011;238:463-467.

11. Goodwin LV, Goggs R, Chan DL, Allenspach K. Hypercoagulability in dogs with protein-losing enteropathy. $J$ Vet Intern Med. 2011 ; 25:273-277.

12. DiBartola SP, Meuten DJ. Renal amyloidosis in two dogs presented for thromboembolic phenomena. J Am Anim Hosp Assoc. 1980;16:129-135.

13. Green RA, Kabel AL. Hypercoagulable state in three dogs with nephrotic syndrome: role of acquired antithrombin III deficiency. J Am Vet Med Assoc. 1982;181:914-917.

14. Burns MG, Kelly AB, Hornof WJ, Howerth EW. Pulmonary artery thrombosis in three dogs with hyperadrenocorticism. J Am Vet Med Assoc. 1981;178:388-393.

15. Vilar Saavedra P, Lara García A, Zaldívar López S, Couto G. Hemostatic abnormalities in dogs with carcinoma: a thromboelastographic characterization of hypercoagulability. Vet J. 201 1;190:e78-e83.

16. Kristensen AT, Wiinberg B, Jessen LR, Andreasen E, Jensen AL. Evaluation of human recombinant tissue factor-activated thromboelastography in 49 dogs with neoplasia. $J$ Vet Intern Med. 2008;22:140-147.

17. Otto C.M., Rieser TM, Brooks MB, Russell MW. Evidence of hypercoagulability in dogs with parvoviral enteritis. J Am Vet Med Assoc. 2000;217: 1500-1504.

18. de Laforcade AM, Freeman LM, Shaw SP, Brooks MB, Rozanski EA, Rush JE. Hemostatic changes in dogs with naturally occurring sepsis. J Vet Intern Med. 2003; 17 : 674-679.
19. Donahue SM, Otto CM. Thrombelastography: a tool for measuring hypercoagulability, hypocoagulability, and fibrinolysis. J Vet Emerg Crit Care. 2005; 15:9-16.

20. Wagg CR, Boysen SR, Bédard C. Thrombelastography in dogs admitted to an intensive care unit. Vet Clin Pathol. 2009;38:453-461.

21. Kupesiz A, Rajpurkar M, Warrier I, et al. Tissue plasminogen activator induced fibrinolysis: standardization of method using thromboelastography. Blood Coagul Fibrinolysis. 2010;21:320-324.

22. Kupesiz OA, Chitlur MB, Hollon W, et al. Fibrinolytic parameters in children with noncatheter thrombosis: a pilot study. Blood Coagul Fibrinolysis. 2010;21:313-319.

23. Gallimore MJ, Harris SL, Tappenden KA, Winter M, Jones DW. Urokinase-induced fibrinolysis in thromboelastography: a model for studying fibrinolysis and coagulation in whole blood. J Thromb Haemost. 2005;3:2506-2513.

24. Nielsen VG, Cohen BM, Cohen E. Elastic modulusbased thrombelastographic quantification of plasma clot fibrinolysis with progressive plasminogen activation. Blood Coagul Fibrinolysis. 2006;17:75-81.

25. Foley JH, Petersen KU, Rea CJ, et al. Solulin increases clot stability in whole blood from individuals and dogs with hemophilia. Blood. 2012;1 19:3622-3628.

26. Summaria L, Sandesara J, Yang G, Vagher JP, Caprini JA. In vitro comparison of fibrinolytic activity of plasminogen activators using a thrombelastographic method: in vivo evaluation of the B-chain-streptokinase complex in the dog model using pre-titered doses. J Thromb Haemost. 1986;56:71-79.

27. Carrieri C, Galasso R, Semeraro F, Ammollo CT, Semeraro N, Colucci M. The role of thrombin activatable fibrinolysis inhibitor and factor XI in platelet-mediated fibrinolysis resistance: a thromboelastographic study in whole blood. J Thromb Haemost. 201 1;9:154-162.

28. Wiinberg B, Kristensen AT. Thromboelastography in veterinary medicine. Semin Thromb Hemost. 2010;36:747-756.

29. Vilar P, Couto CG, Westendorf N, Iazbik C, Charske J, Marín L. Thromboelastographic tracings in retired racing greyhounds and in non-greyhound dogs. J Vet Intern Med. 2008;22:374-379.

30. Saavedra PV, Stingle N, Iazbik C, et al. Thromboelastographic changes after gonadectomy in retired racing greyhounds. Vet Rec. 2011;169:99.

31. Vilar-Saavedra P, Hosoya K. Thromboelastographic profile for a dog with hypocoagulable and hyperfibrinolytic phase of disseminated intravascular coagulopathy. J Small Anim Pract. 201 1;52:656-659.

32. Wiinberg B, Jensen AL, Rojkjaer R, Johansson P, Kjelgaard-Hansen M, Kristensen AT. Validation of 
human recombinant tissue factor-activated thromboelastography on citrated whole blood from clinically healthy dogs. Vet Clin Pathol. 2005;34:389-393.

33. Levi M, van der Poll T, Schultz M. New insights into pathways that determine the link between infection and thrombosis. Neth J Med. 2012;70:1 14-120.

34. Jessen LR, Wiinberg B, Kjelgaard-Hansen M, Jensen AL, Rozanski E, Kristensen AT. Thrombin-activatable fibrinolysis inhibitor activity in healthy and diseased dogs. Vet Clin Pathol. 2010;39:296-301.

35. Donahue S, Brooks M, Otto CM. Examination of hemostatic parameters to detect hypercoagulability in dogs with severe protein-losing nephropathy. $J$ Vet Emerg Crit Care. 2011;21:346-355.
36. Cook AK, Cowgill LD. Clinical and pathological features of protein-losing glomerular disease in the dog: a review of 137 cases (1985-1992). J Am Anim Hosp Assoc. 1996;32:313-322.

37. Marschner CB, Bjørnvad CR, Kristensen AT, Wiinberg B. Thromboelastography results on citrated whole blood from clinically healthy cats depend on modes of activation. Acta Vet Scand. 2010;52:38.

38. Johansson PI, Bochsen L, Andersen S, Viuff D. Investigation of the effect of kaolin and tissue factoractivated whole blood, on clot forming variables, as evaluated by thromboelastography. Transfusion. 2008;48:2377-2382. 